\title{
SOME CHROMATIC PHENOMENA \\ IN THE HOMOTOPY OF MSp
}

\author{
ANDREW BAKER
}

\section{Introduction.}

In this paper, we derive formulæ in Brown-Peterson homology at the prime 2 related to the family of elements $\varphi_{n} \in \mathrm{MSp}_{8 n-3}$ of $\mathrm{N}$. Ray, whose central rôle in the structure of MSp has been highlighted by recent work of $\mathrm{V}$. Vershinin and other Russian topologists. In effect, we give explicit "chromatic" representatives for these elements, which were known to be detected in KO and mod 2 KU-homology, and are thus " $v_{1}$-periodic" in the parlance of [4] and [5]. In future work we will investigate further the $v_{1}$ periodic part of MSp and discuss the relationship of our work with that of B. Botvinnik.

I would like to thank Nigel Ray for many helpful discussions and large amounts of advice on MSp (including severe warnings!) over many years; in particular, $\S 5$ in this paper was prompted by his suggestions about the detection of $\varphi_{n}$ in the classical Adams spectral sequence. I would also like to thank Boris Botvinnik, Vassily Gorbunov and Vladimir Vershinin for discussions on the material of earlier versions of this paper both during and after the J. F. Adams Memorial Symposium and in particular for bringing to my attention Bŭhstaber's article [2] which contains related results.

\section{$\S 1$ Some algebraic results on $E_{*}(\mathrm{MSp})$.}

Let $E$ be a commutative ring spectrum, and let $x^{E} \in E^{2}\left(\mathbb{C P}^{\infty}\right)$ be a complex orientation in the sense of [1]. Then the results of the following Theorem are well known.

\section{Theorem (1.1).}

a) The natural map $j_{1}: \mathbb{C P}^{\infty} \longrightarrow \mathbb{H} \mathrm{P}^{\infty}$ induces a split monomorphism

$$
E^{*}\left(\mathbb{H} \mathrm{P}^{\infty}\right) \stackrel{j_{1}^{*}}{\longrightarrow} E^{*}\left(\mathbb{C} \mathrm{P}^{\infty}\right)
$$

and a split epimorphism

$$
E_{*}\left(\mathbb{C P}^{\infty}\right) \stackrel{j_{1 *}}{\longrightarrow} E_{*}\left(\mathbb{H} \mathrm{P}^{\infty}\right)
$$

of modules over $E_{*}$.

Key words and phrases. Symplectic bordism, Brown-Peterson theory.

The author would like to acknowledge the support of the Science and Engineering Research Council whilst this research was conducted.

Published in 'Adams Memorial Symposium on Algebraic Topology, Vol. 2' editors N. Ray \& G. Walker, CUP (1992), 263-80.

[Version 15: 06/01/2020] 
b) As an $E_{*}$ algebra,

$$
E_{*}(\mathrm{MSp})=E_{*}\left[Q_{k}^{E}: k \geqslant 1\right],
$$

and moreover the natural morphism of ring spectra $j: \mathrm{MSp} \longrightarrow \mathrm{MU}$ induces an embedding of $E_{*}$ algebras

$$
j_{*}: E_{*}(\mathrm{MSp}) \stackrel{j_{*}}{\longrightarrow} E_{*}(\mathrm{MU}) .
$$

We will need explicit sets of generators for the $E$ homology and cohomology of $\mathbb{H P}^{\infty}$ and MSp. Recall the canonical complex line bundle $\eta \longrightarrow \mathbb{C P}^{\infty}$ and its $E$-theory 1st Chern class $c_{1}^{E}(\eta)=x^{E}$; we have $E^{*}\left(\mathbb{C P}^{\infty}\right)=E_{*}\left[\left[x^{E}\right]\right]$. Then the map $j_{1}: \mathbb{C P}^{\infty} \longrightarrow \mathbb{H P}^{\infty}$ classifies the quaternionic line bundle $\eta \otimes_{\mathbb{C}} \mathbb{H} \longrightarrow \mathbb{C P}^{\infty}$, which has as its underlying complex bundle $\eta+\bar{\eta}$, where $\overline{()}$ denotes complex conjugation. We define the E-theory 1st symplectic Pontrjagin class of a symplectic bundle $\zeta \longrightarrow X$ to be the negative of the $E$-theory 2 nd Chern class of the complex bundle $\zeta^{\prime}$ underlying $\zeta$,

$$
\wp^{E}(\zeta)=-c_{2}\left(\zeta^{\prime}\right) \in E^{4}(X)
$$

In particular we set $\wp^{E}=\wp^{E}(\xi) \in E^{4}\left(\mathbb{H} \mathrm{P}^{\infty}\right)$, where $\xi \longrightarrow \mathbb{H} \mathrm{P}^{\infty}$ is the canonical symplectic line bundle; this gives

$$
j_{i}^{*} \wp^{E}=\wp^{E}(\eta+\bar{\eta})=-c_{1}^{E}(\eta) c_{1}^{E}(\bar{\eta}) \in E^{4}\left(\mathbb{C P}^{\infty}\right) .
$$

We also have (as graded algebras over $E_{*}$ )

$$
E^{*}\left(\mathbb{H} \mathrm{P}^{\infty}\right)=E_{*}\left[\left[\wp^{E}\right]\right]
$$

Now let the elements $\beta_{n}^{E} \in E_{2 n}\left(\mathbb{C P}^{\infty}\right), n \geqslant 0$, form the standard $E_{*}$ basis for $E_{*}\left(\mathbb{C P}^{\infty}\right)$ as detailed in [1]; this basis is dual to that of the monomials $\left(x^{E}\right)^{n}$ in $E^{*}\left(\mathbb{C P}^{\infty}\right)$ under the Kronecker pairing $\langle$,$\rangle :$

$$
\left\langle\left(x^{E}\right)^{r}, \beta_{s}^{E}\right\rangle=\delta_{r, s}
$$

where $\delta$ is the Kronecker delta function. Also for any $T$, let $\bar{T}=[-1]_{E}(T)$ be the -1 series for the formal group law $F^{E}(X, Y) \in E_{*}[[X, Y]]$ associated to the orientation $x^{E}$ as described in [1]; notice that $\overline{x^{E}}=c_{1}^{E}(\bar{\eta})$.

Now in $E_{*}\left(\mathbb{H P}^{\infty}\right)$ we can define a sequence of elements $\gamma_{n} \in E_{4 n}\left(\mathbb{H} \mathrm{P}^{\infty}\right), n \geqslant 0$, by requiring that these are dual to the $\left(\wp^{E}\right)^{n}$ :

$$
\left\langle\left(\wp^{E}\right)^{r}, \gamma_{s}\right\rangle=\delta_{r, s}
$$

It is easily verified that a generating function for these elements is the series

$$
\sum_{n \geqslant 0} \gamma_{n}(-T \bar{T})^{n}=\sum_{n \geqslant 0} j_{1 *} \beta_{n}^{E} T^{n} .
$$

Let $i_{1}: \mathbb{H} \mathrm{P}^{\infty} \simeq \operatorname{MSp}(1) \longrightarrow \Sigma^{4} \mathrm{MSp}$ be the standard map, then we have

Theorem (1.5).

a) The elements $\gamma_{n}$ for $n \geqslant 0$ form an $E_{*}$ basis for $E_{*}\left(\mathbb{H} \mathrm{P}^{\infty}\right)$.

b) The elements $i_{1 *} \gamma_{n+1}$ for $n \geqslant 1$ form a set of polynomial generators for the $E_{*}$ algebra $E_{*}(\mathrm{MSp})$. 
From now on we set $Q_{n}^{E}=i_{1 *} \gamma_{n+1} \in E_{4 n}(\mathrm{MSp})$.

We also need some information on the image of the ring homomorphism $j_{*}: E_{*}(\mathrm{MSp}) \longrightarrow E_{*}(\mathrm{MU})$. We have the two generating functions

$$
\begin{aligned}
Q^{E}(T) & =\sum_{n \geqslant 0} Q_{n}^{E} T^{n+1} \in E_{*}(\mathrm{MSp})[[T]], \\
j_{*} Q^{E}(T) & =\sum_{n \geqslant 0} j_{*} Q_{n}^{E} T^{n+1} \in E_{*}(\mathrm{MU})[[T]] .
\end{aligned}
$$

Recall from [1] the standard generators $B_{n}^{E} \in E_{2 n}(\mathrm{MU})$, (for $n \geqslant 0$ and $B_{0}=1$ ) for which

$$
E_{*}(\mathrm{MU})=E_{*}\left[B_{n}^{E}: n \geqslant 1\right],
$$

and let $B^{E}(T)=\sum_{n \geqslant 0} B_{n}^{E} T^{n+1}$. The proof of the next result is left to the reader.

Proposition (1.6). The series $j_{*} Q^{E}(-T \bar{T}) \in E_{*}(\mathrm{MU})$ satisfies the equation

$$
Q^{E}(-T \bar{T})=-B^{E}(T) B^{E}(\bar{T}) \text {. }
$$

\section{$\S 2$ Symplectic Pontrjagin classes in $E \wedge$ MSp theory.}

Recall that the ring spectrum MSp is universal for orientations for quaternionic bundles. The universal orientation is induced from the class

$$
\wp^{\mathrm{MSp}}: \mathbb{H} \mathrm{P}^{\infty} \simeq \operatorname{MSp}(1) \longrightarrow \Sigma^{4} \mathrm{MSp} \in \operatorname{MSp}^{4}\left(\mathbb{H} \mathrm{P}^{\infty}\right) .
$$

We also have

$$
\operatorname{MSp}^{*}\left(\mathbb{H P}^{\infty}\right)=\operatorname{MSp}_{*}\left[\left[\wp^{\mathrm{MSp}}\right]\right] .
$$

Now let $E$ be a complex oriented ring spectrum as in $\S 1$. Then the class $\wp^{E}$ will serve as a universal orientation for quaternionic line bundles in $E$ theory. We can consider the representable cohomology theory $(E \wedge \mathrm{MSp})^{*}()$ on either of the categories of CW spectra or spaces. As $E_{*}(\mathrm{MSp})$ is free over $E_{*}$, we have a Boardman isomorphism

$$
\left(E \wedge \mathrm{MSp}^{*}() \cong E_{*}(\mathrm{MSp}) \widehat{\otimes}_{E_{*}} E_{*}(\text { ) }\right.
$$

where $\widehat{\otimes}$ denotes the completed tensor product with respect to the skeletal topology for infinite complexes. From $\S 1$ and standard arguments about the map $i_{1}: \mathbb{H P}^{\infty} \longrightarrow \Sigma^{4} \mathrm{MSp}$, we have

Proposition (2.3). In $(E \wedge \mathrm{MSp})^{*}\left(\mathbb{H} \mathrm{P}^{\infty}\right) \cong E_{*}(\mathrm{MSp}) \widehat{\otimes}_{E_{*}} E^{*}\left(\mathbb{H} \mathrm{P}^{\infty}\right)$ we have the identity

$$
\wp^{\mathrm{MSp}}=Q^{E}\left(\wp^{E}\right) \text {. }
$$

For later convenience we also introduce the series

$$
N^{E}(T)=\sum_{n \geqslant 0} N_{n}^{E} T^{n+1} \in E_{*}(\mathrm{MSp})[[T]]
$$

determined by the equation

$$
N^{E}\left(Q^{E}(T)\right)=T \text {. }
$$

Notice that $N_{n}^{E} \equiv-Q_{n}^{E}$ modulo decomposables, and hence we can take the $N_{n}^{E}$ to be polynomial generators for $E_{*}(\mathrm{MSp})$. 
$\S 3$ Detecting Ray's element $\varphi_{n}$ with a complex oriented cohomology theory.

In this section we again let $E$ be a complex oriented commutative ring spectrum. We will also require the following assumption to hold:

TF: $\quad E_{*}$ is torsion free.

This condition is satisfied for the following spectra: $E=\mathrm{MU}, \mathrm{BP}, \mathrm{KU}, \mathrm{HZ}$, which include all those which we will be explicitly considering in this paper.

Now consider the space $\mathbb{R} \mathrm{P}^{\infty}$. Let $\rho \longrightarrow \mathbb{R} \mathrm{P}^{\infty}$ be the canonical real line bundle and $\lambda=$ $\rho \otimes_{\mathbb{R}} \mathbb{C} \longrightarrow \mathbb{R P}^{\infty}$ be its complexification. Let $w^{E}=c_{1}^{E}(\lambda) \in E^{2}\left(\mathbb{R P}^{\infty}\right)$. Then we have

$$
E^{*}\left(\mathbb{R P}^{\infty}\right)=E_{*}\left[\left[w^{E}\right]\right] /\left([2]_{E}\left(w^{E}\right)\right)
$$

where $[2]_{E} T$ denotes the 2 series of the formal group law associated to $x^{E}$.

Now we will consider the space $\mathbb{R} \mathrm{P}^{\infty} \times \mathbb{H} \mathrm{P}^{\infty}$ and apply the cohomology theory $(E \wedge \mathrm{MSp})^{*}()$ to it. Since both $E_{*}(\mathrm{MSp})$ and $E^{*}\left(\mathbb{H}^{\infty}\right)$ are free modules over $E_{*}$, we have the following isomorphisms

$$
\begin{aligned}
& \left.(E \wedge \mathrm{MSp})^{*}\left(\mathbb{R P}^{\infty} \times \mathbb{H} \mathrm{P}^{\infty}\right) \cong E_{*}(\mathrm{MSp}) \widehat{\otimes}_{E_{*}} E^{*}(\mathbb{R P})^{\infty}\right) \widehat{\otimes}_{E_{*}} E^{*}\left(\mathbb{H P} \mathrm{P}^{\infty}\right) \\
& (E \wedge \mathrm{MSp})^{*}\left(\mathbb{R P}^{\infty} \times \mathbb{C P} \mathrm{P}^{\infty}\right) \cong E_{*}(\mathrm{MSp}) \widehat{\otimes}_{E_{*}} E^{*}\left(\mathbb{R P} \mathrm{P}^{\infty}\right) \widehat{\otimes}_{E_{*}} E^{*}\left(\mathbb{C P} \mathrm{P}^{\infty}\right)
\end{aligned}
$$

Let $\xi \longrightarrow \mathbb{H} \mathrm{P}^{\infty}$ be the canonical quaternionic line bundle. Then the quaternionic bundle

$$
\rho \otimes_{\mathbb{R}} \xi=\lambda \otimes_{\mathbb{C}} \xi \longrightarrow \mathbb{R} \mathrm{P}^{\infty} \times \mathbb{H} \mathrm{P}^{\infty}
$$

is defined and so has a 1st symplectic Pontrjagin class in each of the theories represented by the spectra MSp, $E$ and $E \wedge$ MSp.

In the ring $(E \wedge \mathrm{MSp})^{*}\left(\mathbb{R} \mathrm{P}^{\infty} \times \mathbb{H} \mathrm{P}^{\infty}\right)$, we have expressions of the form

$$
\wp^{E}\left(\rho \otimes_{\mathbb{R}} \xi\right)=\wp^{E}+w^{2}+\sum_{n \geqslant 1} \widehat{\theta}_{n}^{E}\left(\wp^{E}\right)^{n+1}
$$

and

$$
\wp^{\mathrm{MSp}}\left(\rho \otimes_{\mathbb{R}} \xi\right)=\wp^{\mathrm{MSp}}+w^{2}+\sum_{n \geqslant 1} \widehat{\theta}_{n}^{\mathrm{MSp}}\left(\wp^{\mathrm{MSp}}\right)^{n+1}
$$

where $\widehat{\theta}_{n}^{E} \in E^{4 n}\left(\mathbb{R} \mathrm{P}^{\infty}\right)$ and $\widehat{\theta}_{n}^{\mathrm{MSp}} \in \mathrm{MSp}^{4 n}\left(\mathbb{R} \mathrm{P}^{\infty}\right)$. Upon applying the split monomorphism $j_{1}^{*}$ these yield the following equations in $(E \wedge \mathrm{MSp})^{*}\left(\mathbb{R P}^{\infty} \times \mathbb{C P}^{\infty}\right)$ :

$$
\begin{aligned}
\wp^{E}\left(\left(\lambda \otimes_{\mathbb{C}} \eta\right) \otimes_{\mathbb{C}} \mathbb{H}\right) & =j_{1}^{*}\left[\wp^{E}+w^{2}+\sum_{n \geqslant 1} \widehat{\theta}_{n}^{E}\left(\wp^{E}\right)^{n+1}\right], \\
\wp^{\mathrm{MSp}}\left(\left(\lambda \otimes_{\mathbb{C}} \eta\right) \otimes_{\mathbb{C}} \mathbb{H}\right) & =j_{1}^{*}\left[\wp^{\mathrm{MSp}}+w^{2}+\sum_{n \geqslant 1} \widehat{\theta}_{n}^{\mathrm{MSp}}\left(\wp^{\mathrm{MSp}}\right)^{n+1}\right],
\end{aligned}
$$


Now recall from $(2.3)$ that $\wp^{\mathrm{MSp}}=Q^{E}\left(\wp^{E}\right)$. Thus we obtain

$$
\wp^{\mathrm{MSp}}\left(\left(\lambda \otimes_{\mathbb{C}} \eta\right) \otimes_{\mathbb{C}} \mathbb{H}\right)=Q^{E}\left(j_{1}^{*} \wp^{E}+w^{2}+\sum_{n \geqslant 1} \widehat{\theta}_{n}^{E}\left(j_{1}^{*} \wp^{E}\right)^{n+1}\right) .
$$

Recall from [6] the following construction. Consider the inclusion of the bottom cell $S^{1} \cong \mathbb{R P}^{1} \hookrightarrow \mathbb{R} \mathrm{P}^{\infty}$. Then we can restrict $\rho, \lambda \longrightarrow \mathbb{R} \mathrm{P}^{\infty}$ to $\rho_{1}, \lambda_{1} \longrightarrow \mathbb{R P}^{1}$ and obtain the classes

$$
\wp^{\mathrm{MSp}}\left(\rho_{1} \otimes_{\mathbb{R}} \xi\right) \in \operatorname{MSp}^{4}\left(\mathbb{R P} \mathrm{P}^{1} \times \mathbb{H} \mathrm{P}^{\infty}\right)
$$

and its image under $j_{1}^{*}$,

$$
\wp^{\mathrm{MSp}}\left(\lambda_{1} \otimes_{\mathbb{C}}(\eta+\bar{\eta})\right) \in \operatorname{MSp}^{4}\left(\mathbb{R} \mathrm{P}^{1} \times \mathbb{C P}^{\infty}\right) .
$$

By definition, Ray's elements $\theta_{n} \in \mathrm{MSp}_{4 n-3}$ are given by

$$
\wp^{\mathrm{MSp}}\left(\rho_{1} \otimes_{\mathbb{R}} \xi\right)-\wp^{\mathrm{MSp}}=\sum_{n \geqslant 1} \theta_{n}\left(\wp^{\mathrm{MSp}}\right)^{n}
$$

in $\operatorname{MSp}^{*}\left(\mathbb{R P}^{1} \times \mathbb{H} \mathrm{P}^{\infty}\right)$, and we also have

$$
\wp^{\mathrm{MSp}}\left(\left(\lambda_{1} \otimes_{\mathbb{C}} \eta\right) \otimes_{\mathbb{C}} \mathbb{H}\right)-\wp^{\mathrm{MSp}}=\sum_{n \geqslant 1} \theta_{n}\left(\wp^{\mathrm{MSp}}\right)^{n}
$$

in $(E \wedge \mathrm{MSp})^{*}\left(\mathbb{R} \mathrm{P}^{1} \times \mathbb{C P} \mathrm{P}^{\infty}\right)$. Stably, the smash product $\mathbb{R} \mathrm{P}^{1} \wedge \mathbb{H} \mathrm{P}^{\infty}$ is a retract of $\mathbb{R} \mathrm{P}^{1} \times \mathbb{H} \mathrm{P}^{\infty}$, and this allows us to identify the above expressions with elements of $\operatorname{MSp}^{*}\left(\mathbb{R P}^{1} \wedge \mathbb{H P}^{\infty}\right)$ and $(E \wedge \mathrm{MSp})^{*}\left(\mathbb{R P}^{1} \wedge \mathbb{C P}^{\infty}\right)$. We will also use the notation $\varphi_{n}=\theta_{2 n}$ of $[6]$.

We can factor the inclusion $\mathbb{R P}^{1} \hookrightarrow \mathbb{R} \mathrm{P}^{\infty}$ as $\mathbb{R} \mathrm{P}^{1} \longleftrightarrow \mathbb{R P}^{2} \hookrightarrow \mathbb{R} \mathrm{P}^{\infty}$, and since $\mathbb{R P}^{2}$ is a $\mathbb{Z} / 2$ Moore space, we see that $2 \theta_{n}=0$. Under our assumption $\mathbf{T F}$ together with (3.1), we thus have that $\theta_{n} \longmapsto 0$ under the $E$ theory Hurewicz homomorphism $\mathrm{MSp}_{*} \longrightarrow E_{*}(\mathrm{MSp})$. Equivalently, the class $\wp^{\mathrm{MSp}}\left(\left(\lambda_{1} \otimes_{\mathbb{C}} \eta\right) \otimes_{\mathbb{C}} \mathbb{H}\right)-\wp^{\mathrm{MSp}}$ maps to 0 under the natural homomorphism $\operatorname{MSp}^{*}\left(\mathbb{R} \mathrm{P}^{1} \wedge \mathbb{H} \mathrm{P}^{\infty}\right) \longrightarrow(E \wedge \mathrm{MSp})^{*}\left(\mathbb{R P}^{1} \wedge \mathbb{H} \mathrm{P}^{\infty}\right)$. Let

$$
\begin{aligned}
\wp^{\mathrm{MSp}}\left(\lambda_{2} \otimes_{\mathbb{C}} \xi\right) & =\wp^{\mathrm{MSp}}+\sum_{n \geqslant 1} \tilde{\theta}_{n}\left(\wp^{\mathrm{MSp}}\right)^{n} \\
& \in(E \wedge \mathrm{MSp})^{*}\left(\mathbb{R} \mathrm{P}^{2} \times \mathbb{H} \mathrm{P}^{\infty}\right)
\end{aligned}
$$

where

$$
\widetilde{\theta}_{n} \in \operatorname{MSp}^{*}\left(\mathbb{R P} P^{2} \wedge \mathbb{H} \mathrm{P}^{\infty}\right) \subset(E \wedge \mathrm{MSp})^{*}\left(\mathbb{R} \mathrm{P}^{2} \wedge \mathbb{H} \mathrm{P}^{\infty}\right)
$$

is the image of $\widehat{\theta}^{\mathrm{MSp}}$ under the map induced by the inclusion $\mathbb{R P}^{2} \hookrightarrow \mathbb{R} \mathrm{P}^{\infty}$. Now for any spectrum $F$ with $F_{*}$ torsion free there is an isomorphism $F^{k}\left(\mathbb{R P}^{2}\right) \cong F_{2-k} / 2 F_{2-k}$ induced from Spanier-Whitehead duality. In particular, this applies to the cases $F=E \wedge \mathrm{MSp}$, MSp that we are dealing with, and thus we can interpret $\widetilde{\theta}_{n}$ as an element of $\operatorname{MSp}_{4 n-2} /(2) \subset E_{4 n-2}(\mathrm{MSp}) /(2)$. Let $w_{2} \in E^{2}\left(\mathbb{R P}^{2}\right)$ be the restriction of the generator $w^{E} \in E^{2}\left(\mathbb{R P}^{\infty}\right)$; note that $w_{2}=c_{1}^{E}\left(\lambda_{2}\right)$.

We can now give our main calculational result. 
Theorem (3.4). Let $u=x^{E}$ and $\bar{u}=\overline{x^{E}}$, satisfying

$$
-u \bar{u}=N^{E}\left(j_{1}^{*} \wp^{\mathrm{MSp}}\right)
$$

and

$$
-u \bar{u}, u+\bar{u} \in \operatorname{im}\left[E^{*}\left(\mathbb{H} \mathrm{P}^{\infty}\right) \longrightarrow(E \wedge \mathrm{MSp})^{*}\left(\mathbb{H} \mathrm{P}^{\infty}\right)\right]
$$

Then under assumption TF together with (3.1), we have the following identity in $(E \wedge \mathrm{MSp})_{*}\left(\mathbb{R P}^{2} \wedge \mathbb{C P}^{\infty}\right)$ :

$$
\wp^{\mathrm{MSp}}\left(\left(\left(\lambda_{2} \otimes_{\mathbb{C}} \eta\right) \otimes_{\mathbb{C}} \mathbb{H}\right)\right)+u \bar{u}=\frac{w_{2}}{N^{E^{\prime}}\left(j_{1}^{*} \wp^{\mathrm{MSp}}\right)}\left[\frac{u}{\log ^{E \prime}(\bar{u})}+\frac{\bar{u}}{\log ^{E \prime}(u)}\right],
$$

and moreover this element is equal to $j_{1}^{*} \wp^{\mathrm{MSp}}\left(\rho_{2} \otimes_{\mathbb{R}} \xi\right)-j_{1}^{*} \wp^{\mathrm{MSp}}$ and lies in the image of the natural map $\mathrm{MSp}^{*}\left(\mathbb{R P}^{2} \wedge \mathbb{H} \mathrm{P}^{\infty}\right) \longrightarrow(E \wedge \mathrm{MSp})^{*}\left(\mathbb{R} \mathrm{P}^{2} \wedge \mathbb{H} \mathrm{P}^{\infty}\right)$.

Proof. We need to recall some relevant facts. Firstly, we have

$$
w_{2}^{2}=0=2 w_{2}
$$

Hence only the first order terms in $w_{2}$ are required. Secondly, we have the following formula for formal derivatives:

$$
Q^{E^{\prime}}\left(N^{E}(T)\right)=\frac{1}{N^{E \prime}(T)}
$$

Finally, by definition,

$$
\wp^{E}\left(\left(\lambda_{2} \otimes_{\mathbb{C}} \eta\right) \otimes_{\mathbb{C}} \mathbb{H}\right)=-c^{E}\left(\lambda_{2} \otimes_{\mathbb{C}} \eta+\lambda_{2} \otimes_{\mathbb{C}} \bar{\eta}\right)
$$

But expanding this using the Cartan formula, we obtain

$$
\begin{aligned}
\wp^{E}\left(\left(\lambda_{2} \otimes_{\mathbb{C}} \eta\right) \otimes_{\mathbb{C}} \mathbb{H}\right) & =-c_{1}^{E}\left(\lambda_{2} \otimes_{\mathbb{C}} \eta\right) c_{1}^{E}\left(\lambda_{2} \otimes_{\mathbb{C}} \bar{\eta}\right) \\
& =-F^{E}\left(w_{2}, u\right) F^{E}\left(\bar{w}_{2}, \bar{u}\right) \\
& =-F^{E}\left(w_{2}, u\right) F^{E}\left(w_{2}, \bar{u}\right)
\end{aligned}
$$

using the fact that $\lambda_{2}^{2}$ is a trivial line bundle. In this, we are setting $u=c_{1}^{E}(\eta)$ and $\bar{u}=c_{1}^{E}(\bar{\eta})$.

We also require the following well known Lemma.

Let $\log ^{E}(X) \in E \otimes \mathbb{Q}[[X]]$ denote the logarithm series of the formal group law $F^{E}$.

Lemma (3.5). The formula

$$
\log ^{E \prime}(X)=\frac{1}{F_{2}^{E}(X, 0)}
$$

holds in $E_{*}[[X]]$, where $F_{2}^{E}(X, Y)=\frac{\partial}{\partial Y} F^{E}(X, Y)$. Hence the logarithm of $F^{E}, \log ^{E}(X)$, has coefficients in $E_{*}$.

Proposition (3.4) now follows from these observations. 
As a sample application of this, we consider the case of $E=\mathrm{KU}$, i.e., complex KU-theory. This allows us to determine the KO-theory Hurewicz images of Ray's elements $\theta_{n} \in \mathrm{MSp}_{8 n-3}$. Recall that we have $\mathrm{KU}_{*}=\mathbb{Z}\left[t, t^{-1}\right]$, where $t \in \mathrm{KU}_{2}$ is the Bott generator; this theory clearly satisfies the condition TF. Corresponding to the standard complex orientation in $\mathrm{KU}^{*}($ ), the formal group law $F^{\mathrm{KU}}(X, Y)=X+Y+t X Y$; this has as its logarithm the series.

$$
\log ^{\mathrm{KU}}(X)=t^{-1} \ln (1+t X)=\sum_{k \geqslant 1} \frac{(-t)^{k-1} X^{k}}{k}
$$

Thus we have

$$
\log ^{\mathrm{KU}}(X)=\frac{1}{(1+t X)}
$$

Now the formula of (3.4) becomes

$$
\wp^{\mathrm{MSp}}\left(\left(\left(\lambda_{2} \otimes_{\mathbb{C}} \eta\right) \otimes_{\mathbb{C}} \mathbb{H}\right)\right)=\wp^{\mathrm{MSp}}+\frac{w_{2}}{N^{\mathrm{KU} \prime}\left(\wp^{\mathrm{MSp}}\right)}\left[\frac{u}{\log ^{\mathrm{KU} \prime}(\bar{u})}+\frac{\bar{u}}{\log ^{\mathrm{KU} \prime}(X)}\right]
$$

We also have

$$
[-1]_{\mathrm{KU}}(X)=\frac{-X}{(1+t X)}
$$

Thus we obtain

$$
\begin{aligned}
& \wp^{\mathrm{MSp}}\left(\left(\left(\lambda_{2} \otimes_{\mathbb{C}} \eta\right) \otimes_{\mathbb{C}} \mathbb{H}\right)\right)= \\
& \wp^{\mathrm{MSp}}+\frac{w_{2}}{N^{\mathrm{KU}}\left(\wp^{\mathrm{MSp}}\right)}[u(1+t \bar{u})+\bar{u}(1+t u)] \\
&= \wp^{\mathrm{MSp}}+\frac{w_{2}}{N^{\mathrm{KU} \prime}\left(\wp^{\mathrm{MSp}}\right)} \cdot \frac{-t u^{2}}{(1+t u)} \\
&= \wp^{\mathrm{MSp}}+\frac{t w_{2} \wp^{\mathrm{KU}}}{N^{\mathrm{KU}}\left(\wp^{\mathrm{MSp}}\right)} \\
&= \wp^{\mathrm{MSp}}+\frac{t w_{2} N^{\mathrm{KU}}\left(\wp^{\mathrm{MSp}}\right)}{N^{\mathrm{KU}}\left(\wp^{\mathrm{MSp}}\right)} .
\end{aligned}
$$

The coefficient of $w_{2}\left(\wp^{\mathrm{MSp}}\right)^{n}$ in this series is $\widetilde{\theta}_{n}$. Notice that we can write

$$
\frac{N\left(\wp^{\mathrm{MSp}}\right)}{N^{\mathrm{KU} \prime}\left(\wp^{\mathrm{MSp}}\right)} \equiv \wp^{\mathrm{MSp}}+\frac{\sum_{r \geqslant 1} N_{2 r-1}\left(\wp^{\mathrm{MSp}}\right)^{2 r}}{\sum_{s \geqslant 0} N_{2 s}\left(\wp^{\mathrm{MSp}}\right)^{2 s}} \quad(\bmod 2) .
$$

From this result, we can immediately deduce that $\widetilde{\theta}_{2 r-1}=0$ if $r>1$. This suffices to prove the following result which was conjectured in [6] and also follows from an unpublished result of F. Roush which actually shows that $\theta_{2 r-1}=0$ when $r>1$. Let $\underline{\text { ko }}: \operatorname{MSp} \rightarrow \mathrm{KO}_{*}(\mathrm{MSp})$ be the KO-theory Hurewicz homomorphism. 
Proposition (3.7). For $r>1$, we have in $\mathrm{KO}_{8 r-7}(\mathrm{MSp})$,

$$
\underline{\mathrm{ko}}\left(\theta_{2 r-1}\right)=0 \text {. }
$$

Proof. Let $M(2)=S^{0} \cup_{2} \mathrm{e}^{1}$ denote the mod 2 Moore spectrum and let $\eta: S^{1} \rightarrow S^{0}$ denote the non-trivial element of $\pi_{1}^{S}$ together with the map $S^{1} \wedge \mathrm{KO} \rightarrow \mathrm{KO}$ it induces; let $M(\eta)=S^{0} \cup_{\eta} \mathrm{e}^{2}$ be the mapping cone. Recall from [1] "the Theorem of Reg Wood": there is a cofibre sequence

$$
S^{1} \wedge \mathrm{KO} \stackrel{\eta}{\rightarrow} \mathrm{KO} \rightarrow \mathrm{KO} \wedge M(\eta) \simeq \mathrm{KU} .
$$

Now consider the exact diagram of abelian groups in which the rows are induced from the cofibre sequence for $\eta$ and the columns from the cofibre sequence for multiplication by 2 :

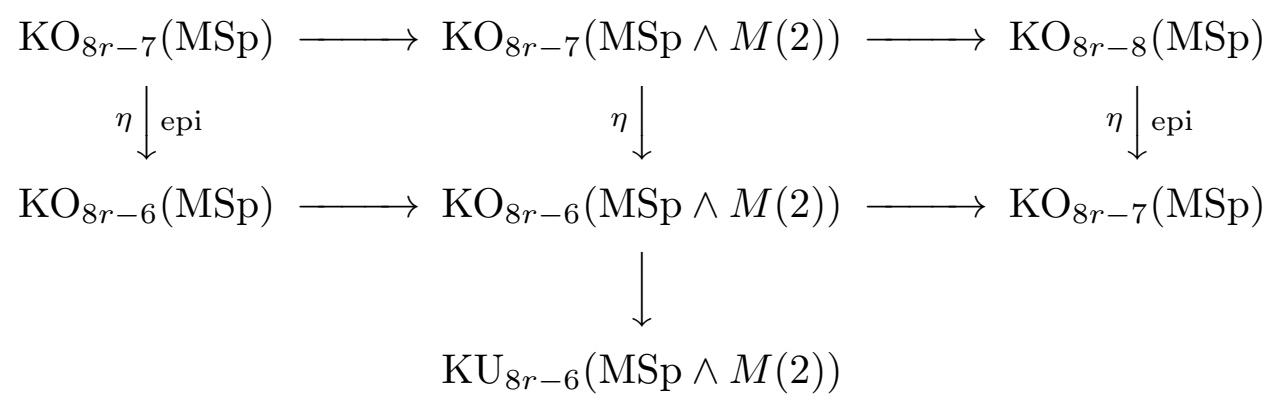

Now $\widetilde{\theta}_{2 r-1}=0$ in the group $\mathrm{KU}_{8 r-6}(\mathrm{MSp} \wedge M(2))$ appearing in this diagram, and therefore we can lift $\underline{\mathrm{ko}}\left(\theta_{2 r-1}\right)$ in the group $\mathrm{KO}_{8 r-7}(\mathrm{MSp})$ to an element of the torsion group $\mathrm{KO}_{8 r-7}(\mathrm{MSp} \wedge M(2))$. But this means that $\mathrm{ko}\left(\theta_{2 r-1}\right)$ is the image of an element of $\mathrm{KO}_{8 r-8}(\mathrm{MSp})$, a torsion free group. The only way that both of these can be true is if $\underline{\mathrm{ko}}\left(\theta_{2 r-1}\right)=0$.

We can also see that for each $n \geqslant 1$,

$$
\widetilde{\varphi}_{n}=\widetilde{\theta}_{2 n} \equiv t N_{2 n-1} \quad(\bmod \text { decomposables }) .
$$

This of course shows that in the ring $\mathrm{KU}_{*}(\mathrm{MSp}) /(2)$, the elements $\widetilde{\varphi}_{n}$ are algebraically independent over $\mathrm{KU}_{*} /(2)$.

$\S 4$ Some calculations in $\mathrm{BP}_{*}(\mathrm{MSp})$.

We now consider the case of $E=\mathrm{BP}$, the Brown-Peterson spectrum at the prime 2; we will assume the reader to be familiar with [1] and [5] which contains detailed information on BP.

We begin with some algebraic Lemmas on the formal group law for BP. Recall that the logarithm of $F^{\mathrm{BP}}$ is the series

$$
\log ^{\mathrm{BP}}(X)=\sum_{n \geqslant 0} \ell_{n} X^{2^{n}} \in\left(\mathrm{BP}_{*} \otimes \mathbb{Q}\right)[[X]],
$$

where $\ell_{n} \in \mathrm{BP}_{2\left(2^{n}-1\right)} \otimes \mathbb{Q}$. The Hazewinkel generators are then defined recursively by the formula

$$
v_{n}=2 \ell_{n}-\sum_{1 \leqslant k \leqslant n-1} \ell_{k} v_{n-k}^{2^{k}} \text { for } n \geqslant 1
$$

We also have the following relation for the 2 -series $[2]_{\mathrm{BP}}(X)$ :

$$
[2]_{\mathrm{BP}}(X) \equiv \sum_{n \geqslant 1}^{\mathrm{BP}} v_{n} X^{2^{n}} \quad(\bmod 2),
$$

where as usual the symbol $\sum^{\mathrm{BP}}$ indicates formal group summation. We will need the following well known facts about the formal derivative of the logarithm. The next result may be known to others but we know of no reference. 
Lemma (4.4). We have the following congruence in $\mathrm{BP}_{*}[[X]]$ :

$$
\log ^{\mathrm{BP}}(X) \equiv \sum_{n \geqslant 0} v_{1}^{2^{n}-1} X^{2^{n}-1} \quad(\bmod 2) .
$$

Proof. Let

$$
\log ^{\mathrm{BP} \prime}(X)=\sum_{n \geqslant 0} L_{n} X^{2^{n}-1}
$$

where $L_{n}=2^{n} \ell_{n}$ is an element of $\mathrm{BP}_{2^{n+1}-2}$. We will show by induction upon $n$ that

$$
L_{n} \equiv v_{1}^{2^{n}-1} \quad(\bmod 2) .
$$

For $n=0$ and 1 , the result is clear. Suppose that it holds for some $n$. Then we have

$$
\begin{aligned}
2^{n} v_{n+1} & =L_{n+1}-\sum_{1 \leqslant k \leqslant n} 2^{n-k} L_{k} v_{n+1-k}^{2^{k}} \\
& \equiv v_{1}^{2^{n+1}-1}(\bmod 2) \quad \text { by the inductive hypothesis. }
\end{aligned}
$$

Hence, the desired result holds for $n+1$ and the Lemma follows.

Our next result allows us to estimate the series $[-1]_{\mathrm{BP}}(X)$ modulo 2. Again it is quite possibly known to others.

Lemma (4.5). The following congruences hold in $\mathrm{BP}_{*}[[X]]$ :

$$
\begin{aligned}
{[-1]_{\mathrm{BP}}(X) } & \equiv \sum_{n \geqslant 0}^{\mathrm{BP}}\left[2^{n}\right]_{\mathrm{BP}}(X)(\bmod 2) \\
& \equiv X+{ }_{\mathrm{BP}} \sum_{\substack{n \geqslant 1 \\
r_{1}, \ldots, r_{n} \geqslant 1}}^{\mathrm{BP}} v_{r_{1}} v_{r_{2}}^{2^{r_{1}}} \cdots v_{r_{2}}^{2^{r_{1}+\cdots+r_{n-1}}} X^{2^{r_{1}+\cdots+r_{n}}}(\bmod 2),
\end{aligned}
$$

where in each case the right hand side is an $X$-adically convergent series.

Proof. The sequence $\left[2^{n}-1\right]_{\mathrm{BP}}(X)$ is Cauchy with respect to the $X$-adic topology on $\mathrm{BP}_{*} /(2)[[X]]$ since the formal group differences of successive terms have the form $\left[2^{n}\right]_{\mathrm{BP}}(X)$ and by (4.3) the leading term of this is that in $X^{2^{n}}$. Hence, we see that the limit of this sequence is

$$
\sum_{n \geqslant 0}^{\mathrm{BP}}\left[2^{n}\right]_{\mathrm{BP}}(X),
$$

giving the desired result.

We are now in a position to calculate $\wp^{\mathrm{MSp}}\left(\left(\left(\lambda_{2} \otimes_{\mathbb{C}} \eta\right) \otimes_{\mathbb{C}} \mathbb{H}\right)\right)$ as an element of $(\mathrm{BP} \wedge$ $\mathrm{MSp})^{*}\left(\mathbb{R} \mathrm{P}^{2} \times \mathbb{H} \mathrm{P}^{\infty}\right)$. Recall that we have from $\S 3$,

$$
\wp^{\mathrm{MSp}}\left(\left(\left(\lambda_{2} \otimes_{\mathbb{C}} \eta\right) \otimes_{\mathbb{C}} \mathbb{H}\right)\right)=\wp^{\mathrm{MSp}}+\frac{w_{2}}{N^{\mathrm{BP} \prime}\left(\wp^{\mathrm{MSp}}\right)}\left[\frac{u}{\log ^{\mathrm{BP} \prime}(\bar{u})}+\frac{\bar{u}}{\log ^{\mathrm{BP} \prime}(X)}\right] .
$$

The ring of power series in $u$ and $\bar{u}$ which is symmetric with respect to the automorphism interchanging these two elements is easily seen to be the power series ring on $-u \bar{u}$, hence we can express this last quantity as an element of $(\mathrm{BP} \wedge \mathrm{MSp})^{*}\left(\mathbb{R P}^{2}\right)\left[\left[\wp^{\mathrm{BP}}\right]\right]$ by making use of the fact that $\wp^{\mathrm{BP}}=-u \bar{u}$. Of course, this will be hard to do explicitly in general, but we can extract sufficient information from our formula to enable us to make some interesting deductions. Amongst these we have the following, which we leave the reader to verify. Recall that in $\mathrm{BP}_{*}$, the ideal $I_{r}=\left(v_{0}, v_{1}, \ldots, v_{r}\right)$ is invariant and prime (here as usual we set $v_{0}=2$ ). 
Theorem (4.6). For $r \geqslant 1$ we have in $(\mathrm{BP} \wedge \mathrm{MSp})^{*}\left(\mathbb{R P}^{2} \times \mathbb{H} \mathrm{P}^{\infty}\right)$ the congruence:

$$
\begin{array}{r}
\wp^{\mathrm{MSp}}\left(\left(\left(\lambda_{2} \otimes_{\mathbb{C}} \eta\right) \otimes_{\mathbb{C}} \mathbb{H}\right)\right) \equiv \wp^{\mathrm{MSp}}+\frac{w_{2}}{N^{\mathrm{BP} \prime}\left(\wp^{\mathrm{MSp}}\right)}\left[v_{r}\left(\wp^{\mathrm{MSp}}\right)^{2^{r-1}}+v_{r+1}\left(\wp^{\mathrm{MSp}}\right)^{2^{r}}\right] \\
\left(\bmod I_{r}+\left(\wp^{\mathrm{MSp}}\right)^{2^{r}+1}\right) .
\end{array}
$$

This allows us to deduce that

$$
\begin{aligned}
& \widetilde{\varphi}_{1}=v_{2}+v_{1} N_{1} \\
& \widetilde{\varphi}_{2}=v_{3}+v_{2} N_{2} \bmod I_{2}+\text { decomposables }
\end{aligned}
$$

and in general

$$
\widetilde{\varphi}_{2^{r-1}}=v_{r+1}+v_{r} N_{2^{r-1}} \bmod I_{r-1}+\text { decomposables, }
$$

where the term "decomposables" refers to decomposables in the $\mathrm{BP}_{*}$ algebra $\mathrm{BP}_{*}\left[N_{k}: k \geqslant 1\right]$.

\section{$\S 5$ Detecting Ray's elements $\varphi_{n}$ in mod 2 homology.}

In this section we will give a formula for the element in the group

$$
\operatorname{Ext}_{\mathcal{A}_{*}}^{14 m-2}\left(\mathbb{F}_{2}, \mathrm{H}_{\mathbb{F}_{2 *}}(\mathrm{MSp})\right)
$$

(a part of the $\mathrm{E}_{2}$ term of the classical Adams Spectral Sequence for $\pi_{*}(\mathrm{MSp})$ ) which detects N. Ray's element $\theta_{m} \in \mathrm{MSp}_{4 m-3}$; here, $\mathcal{A}_{*}$ denotes the dual Steenrod algebra at the prime 2, and $\mathrm{HF}_{2}$ is $\bmod 2$ ordinary homology. For the case of $m=2 n$, we have $\varphi_{n}=\theta_{2 n}$. Our approach is to use the formulæ we obtained in $\S 4$ to determine the image of $\widetilde{\theta}_{m}$ under the composition

$$
\begin{aligned}
\operatorname{Ext}_{\mathrm{BP}_{*}(\mathrm{BP})}^{0 *}\left(\mathrm{BP}_{*}, \mathrm{BP}_{*}(\mathrm{MSp}) /(2)\right) \stackrel{\delta}{\rightarrow} & \\
& \operatorname{Ext}_{\mathrm{BP}_{*}(\mathrm{BP})}^{1 *}\left(\mathrm{BP}_{*}, \mathrm{BP}_{*}(\mathrm{MSp})\right) \stackrel{\rho}{\rightarrow} \operatorname{Ext}_{\mathcal{A}_{*}^{*}}^{1 *}\left(\mathbb{F}_{2}, \mathrm{HF}_{2 *}(\mathrm{MSp})\right),
\end{aligned}
$$

in which $\delta$ denotes the coboundary (of bidegree $(1,0)$ ) induced from the exact sequence of comodules

$$
0 \longrightarrow \mathrm{BP}_{*}(\mathrm{MSp}) \stackrel{\times 2}{\longrightarrow} \mathrm{BP}_{*}(\mathrm{MSp}) \rightarrow \mathrm{BP}_{*}(\mathrm{MSp}) /(2) \longrightarrow 0,
$$

and $\rho$ denotes the reduction map which has bidgree $(0,0)$ and is induced by the morphism of comodules

$$
\left(\mathrm{BP}_{*}(\mathrm{BP}), \mathrm{BP}_{*}(\mathrm{MSp})\right) \longrightarrow\left(\mathrm{HF}_{2 *}\left(\mathrm{HF}_{2}\right)=\mathcal{A}_{*}, \mathrm{HF}_{2 *}(\mathrm{MSp})\right)
$$

From $\S 4$, we have as the generating function of the $\widetilde{\theta}_{m}$ the series

$$
\begin{aligned}
\tilde{\theta}\left(\wp^{\mathrm{MSp}}\right) & =\sum_{m \geqslant 1} \tilde{\theta}_{m}\left(\wp^{\mathrm{MSp}}\right)^{m} \\
& =\frac{1}{N^{\mathrm{BP} \prime}\left(\wp^{\mathrm{MSp}}\right)}\left[\frac{u}{\log ^{\mathrm{BP} \prime}(\bar{u})}+\frac{\bar{u}}{\log ^{\mathrm{BP}}(u)}\right]
\end{aligned}
$$


with the notational conventions of our earlier sections. The coefficients of this series in $\wp^{\mathrm{MSp}}$ lie in $\mathrm{Ext}_{\mathrm{BP} *(\mathrm{BP})}^{0 *}\left(\mathrm{BP}_{*}, \mathrm{BP}_{*}(\mathrm{MSp}) /(2)\right)$, and our first task is to calculate the series whose coefficients are the elements

$$
\psi\left(\widetilde{\theta}_{m}\right) \in \mathrm{BP}_{*}(\mathrm{BP}) \otimes_{\mathrm{BP}_{*}} \mathrm{BP}_{*}(\mathrm{MSp}) /(2) \quad\left(\bmod I^{2}\right)
$$

where $\psi: \mathrm{BP}_{*}(\mathrm{MSp}) \longrightarrow \mathrm{BP}_{*}(\mathrm{BP}) \otimes_{\mathrm{BP}_{*}} \mathrm{BP}_{*}(\mathrm{MSp})$ is the (left) coaction. To do this, we view the above series as lying in the ring $(\mathrm{BP} \wedge \mathrm{MSp})^{*}\left(\mathbb{H P}^{\infty}\right) /(2)$ and the coaction as being a ring homomorphism

$$
\begin{aligned}
&(\mathrm{BP} \wedge \mathrm{MSp})^{*}\left(\mathbb{H} \mathrm{P}^{\infty}\right) \stackrel{\psi}{\rightarrow}(\mathrm{BP} \wedge \mathrm{BP} \wedge \mathrm{MSp})^{*}\left(\mathbb{H} \mathrm{P}^{\infty}\right) \\
& \quad \mathrm{BP}_{*}(\mathrm{BP}) \otimes_{\mathrm{BP}_{*}}(\mathrm{BP} \wedge \mathrm{MSp})^{*}\left(\mathbb{H P}^{\infty}\right) .
\end{aligned}
$$

We view the two factors of $\mathrm{BP}$ in the latter object as left $(L)$ and right $(R)$ indexed by these letters. Then we obtain

$$
\begin{aligned}
& \psi \widetilde{\theta}\left(\wp^{\mathrm{MSp}}\right)=\sum_{m \geqslant 1} \widetilde{\theta}_{m}\left(\wp^{\mathrm{MSp}}\right)^{m}
\end{aligned}
$$

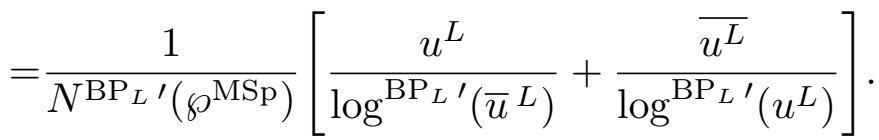

But now in $(\mathrm{BP} \wedge \mathrm{BP})^{*}\left(\mathbb{C P}^{\infty}\right)$, the left $x^{\mathrm{BP}_{L}}$ and right orientations $x^{\mathrm{BP}_{R}}$ are related by the following formula to be found in [5]:

$$
\begin{aligned}
x^{\mathrm{BP}_{L}} & =\sum_{k \geqslant 0}^{\mathrm{BP}_{L}} t_{k}\left(x^{\mathrm{BP}_{R}}\right)^{2^{k}} \\
& \equiv \sum_{k \geqslant 0} t_{k}\left(x^{\mathrm{BP}_{R}}\right)^{2^{k}}(\bmod I) .
\end{aligned}
$$

Hence we have

$$
u^{L}=\sum_{k \geqslant 0}^{\mathrm{BP}_{L}} t_{k}\left(u^{R}\right)^{2^{k}} .
$$

Now we also need to estimate $\log ^{\mathrm{BP}}(X)$ modulo $I^{2}$, where

$$
I=\left(v_{k}: k \geqslant 0\right) \triangleleft \mathrm{BP}_{*}
$$

is the ideal generated by all the $v_{k}$.

Proposition (5.2). In the ring $\mathrm{BP}_{*}[[X]]$ we have the congruence

$$
\log ^{\mathrm{BP}}(X) \equiv 1+v_{1} X \quad\left(\bmod I^{2}\right) .
$$

Proof. We proceed as in the proof of Lemma (4.4) and use the same notation. We must show that for $n \geqslant 2$, we have

$$
L_{n} \equiv 0 \quad\left(\bmod I^{2}\right)
$$


For $n=2$, this is an easy consequence of the formula

$$
2 v_{2}=L_{2}-v_{1}^{3}
$$

defining the Hazewinkel generator $v_{2}$. An induction on $n$ now gives general result.

At this stage we have established that

$$
\begin{aligned}
\psi \widetilde{\theta}\left(\wp^{\mathrm{MSp}}\right) \equiv \frac{1}{N^{\mathrm{BP}_{L} \prime}\left(\wp^{\mathrm{MSp}}\right)}\left[\frac{u^{L}}{\left(1+v_{1} \overline{u^{L}}\right)}+\frac{\overline{u^{L}}}{\left(1+v_{1} u^{L}\right)}\right] \equiv \\
\frac{1}{N^{\mathrm{BP}_{L}{ }^{\prime}\left(\wp^{\mathrm{MSp}}\right)}}\left[\frac{u^{L}}{\left(1+v_{1} \sum_{k \geqslant 0} t_{k}\left(\overline{u^{R}}\right)^{2^{k}}\right)}+\frac{\overline{u^{L}}}{\left(1+v_{1} \sum_{k \geqslant 0} t_{k}\left(u^{R}\right)^{2^{k}}\right)}\right]
\end{aligned}
$$

where the second congruence is a consequence of (5.1). Notice that we have

$$
\begin{aligned}
& \psi \widetilde{\theta}\left(\wp^{\mathrm{MSp}}\right) \equiv \\
& \quad \frac{1}{N^{\mathrm{BP}_{L} \prime}\left(\wp^{\mathrm{MSp}}\right)}\left[\frac{\sum_{k \geqslant 0} t_{k}\left(u^{R}\right)^{2^{k}}}{\left(1+v_{1} \sum_{k \geqslant 0} t_{k}\left(u^{R}\right)^{2^{k}}\right)}+\frac{\sum_{k \geqslant 0} t_{k}\left(\overline{u^{R}}\right)^{2^{k}}}{\left(1+v_{1} \sum_{k \geqslant 0} t_{k}\left(u^{R}\right)^{2^{k}}\right)}\right]
\end{aligned}
$$

since

$$
\bar{X} \equiv X+\sum_{k \geqslant 0} v_{k} X^{2^{k}}\left(\bmod I^{2}\right)
$$

by $(4.5)$.

We still need to deal with the term $N^{\mathrm{BP}_{L}{ }^{\prime}}\left(\wp^{\mathrm{MSp}}\right)$, which has to be expressed in terms of

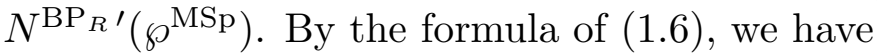

$$
\begin{aligned}
Q^{\mathrm{BP}}\left(T^{2}\right) & \equiv Q^{\mathrm{BP}}(-T \bar{T}) \\
& \equiv B^{\mathrm{BP}}(T)^{2}(\bmod I) \\
& \equiv \sum_{k \geqslant 0} B_{k}^{\mathrm{BP} 2} T^{2 k+2}(\bmod I)
\end{aligned}
$$

in the ring $\mathrm{BP}_{*}(\mathrm{MU})[[T]]$. The coaction on this series is given by

$$
\psi\left(Q^{\mathrm{BP}}\left(T^{2}\right)\right)=\sum_{k \geqslant 0} B^{\mathrm{BP}, \mathrm{BP}}(T)^{2 k+2} \otimes_{\mathrm{BP}_{*}} B_{k}^{\mathrm{BP} 2}
$$

where the series $B^{\mathrm{BP}, \mathrm{BP}}(T) \in \mathrm{BP}_{*}(\mathrm{BP})[[T]]$ is composition inverse of

$$
\sum_{k \geqslant 0}^{\mathrm{BP}} t_{k} T^{2^{k}} \equiv \sum_{k \geqslant 0} t_{k} T^{2^{k}} \quad(\bmod I) .
$$


As $N^{\mathrm{BP}}(Z)$ is the composition inverse of $Q^{\mathrm{BP}}(Z)$, we have

$$
\psi\left(N^{\mathrm{BP}}(Z)\right)=\sum_{k \geqslant 0} t_{k}^{2} \otimes\left(N^{\mathrm{BP}}(Z)\right)^{2^{k}} \quad(\bmod I)
$$

and hence

$$
\psi\left(N^{\mathrm{BP} \prime}(Z)\right)=N^{\mathrm{BP} \prime}(Z) \quad(\bmod I) .
$$

Using this result together with (5.4), we have

$$
\begin{aligned}
\psi \widetilde{\theta}\left(\wp^{\mathrm{MSp}}\right) \equiv & \frac{1}{N^{\mathrm{BP}_{R^{\prime}}\left(\wp^{\mathrm{MSp}}\right)}}\left[\frac{u^{L}}{\left(1+v_{1} \sum_{k \geqslant 0} t_{k}\left(u^{R}\right)^{2^{k}}\right)}+\frac{\overline{u^{L}}}{\left(1+v_{1} \sum_{k \geqslant 0} t_{k}\left(u^{R}\right)^{2^{k}}\right)}\right]
\end{aligned}
$$

$\left(\bmod I^{2}\right)$

Expanding carefully the term inside the square brackets yields

$$
u^{L}+\overline{u^{L}} \equiv \sum_{k \geqslant 0} v_{k}\left(u^{R}\right)^{2^{k}} \quad\left(\bmod I^{2}\right)
$$

and therefore

$$
\psi \widetilde{\theta}\left(\wp^{\mathrm{MSp}}\right) \equiv \frac{\sum_{k \geqslant 0} v_{k}\left(N^{\mathrm{BP}}\left(u^{R}\right)\right)^{2^{k}}}{N^{\mathrm{BP}_{R} \prime}\left(\wp^{\mathrm{MSp}}\right)} \quad\left(\bmod I^{2}\right)
$$

since $N^{\mathrm{BP}}{ }_{R}\left(\wp^{\mathrm{MSp}}\right)=\wp^{\mathrm{BP}}{ }_{R} \equiv\left(u^{R}\right)^{2} \bmod I^{2}$.

Having calculated this, we are at last in a position to determine

$$
\delta\left(\widetilde{\theta}_{m}\right)=\left[\frac{1}{2}(\psi-1 \otimes \operatorname{Id})\left(\widetilde{\theta}_{m}\right)\right] \quad(\bmod I)
$$

We need one further fact, namely that the right unit on the $v_{n}$ has the form

$$
\eta_{R}\left(v_{n}\right) \equiv \sum_{0 \leqslant k \leqslant n} v_{k} t_{n-k}^{p^{k}} \quad\left(\bmod I^{2}\right)
$$

We therefore obtain the following generating function:

$$
\sum_{m \geqslant 1} \delta\left(\widetilde{\theta}_{m}\right)\left(\wp^{\mathrm{MSp}}\right)^{m}=\frac{\sum_{k \geqslant 0} t_{k}\left(u^{R}\right)^{2^{k}}}{1 \otimes N^{\mathrm{BP} \prime}\left(\wp^{\mathrm{MSp}}\right)} \quad(\bmod I)
$$

which when pushed into $\mathcal{A}_{*} \otimes \mathrm{HF}_{2 *}(\mathrm{MSp})$ yields

$$
\frac{\sum_{k \geqslant 0} \zeta_{k}^{2} \otimes N^{\mathrm{H} \mathbb{F}_{2}}\left(\wp^{\mathrm{MSp}}\right)^{2^{k}}}{1 \otimes N^{\mathrm{H} \mathbb{F}_{2} \prime}\left(\wp^{\mathrm{MSp}}\right)},
$$

where $\mathcal{A}_{*}=\mathbb{F}_{2}\left[\zeta_{k}: k \geqslant 1\right]$ with $\zeta_{k}$ being the conjugate of Milnor's generator $\xi_{k} \in \mathcal{A}_{2^{k}-1}$. 
The element $\theta_{m}$ is detected in the classical mod 2 Adams spectral sequence for $\pi_{*}(\mathrm{MSp})$ by an element which originates in the $\mathrm{E}_{2}$ term in the group

$$
\operatorname{Ext}_{\mathcal{A}_{*}}^{14 m-2}\left(\mathbb{F}_{2}, \mathrm{HF}_{2 *}(\mathrm{MSp})\right) \text {. }
$$

For low even values of $m$, these detecting elements are given in the following Table, obtained using the symbolic algebra package MAPLE. Note that $\theta_{1}=\left[\zeta_{1}^{2} \otimes 1\right]$ and $\theta_{2 n}=\varphi_{n}$. Of course, for $n \geqslant 1, \theta_{2 n+1}$ is zero and hence detected by 0 ! The notation is that implied by the cobar construction.

\section{TABLE}

$$
\begin{aligned}
& \varphi_{1}: {\left[\zeta_{1}^{2} \otimes N_{1}+\zeta_{2}^{2} \otimes 1\right] } \\
& \varphi_{2}: {\left[\zeta_{1}^{2} \otimes N_{1} N_{2}+\zeta_{1}^{2} \otimes N_{3}+\zeta_{2}^{2} \otimes N_{2}+\zeta_{2}^{2} \otimes N_{1}^{2}+\zeta_{3}^{2} \otimes 1\right] } \\
& \varphi_{3}:\left[\zeta_{1}^{2} \otimes N_{1} N_{4}+\zeta_{1}^{2} \otimes N_{1} N_{2}^{2}+\zeta_{1}^{2} \otimes N_{5}+\zeta_{1}^{2} \otimes N_{3} N_{2}\right. \\
&\left.\quad+\zeta_{2}^{2} \otimes N_{1}^{2} N_{2}+\zeta_{2}^{2} \otimes N_{4}+\zeta_{3}^{2} \otimes N_{2}\right] \\
& \varphi_{4}:\left[\zeta_{1}^{2} \otimes N_{1} N_{6}+\zeta_{1}^{2} \otimes N_{1} N_{2}^{3}+\zeta_{1}^{2} \otimes N_{5} N_{2}+\zeta_{1}^{2} \otimes N_{3} N_{4}+\zeta_{1}^{2} \otimes N_{3} N_{2}^{2}\right. \\
& \quad+\zeta_{1}^{2} \otimes N_{7} \\
& \quad+\zeta_{2}^{2} \otimes N_{6}+\zeta_{2}^{2} \otimes N_{1}^{2} N_{4}+\zeta_{2}^{2} \otimes N_{1}^{2} N_{2}^{2}+\zeta_{2}^{2} \otimes N_{3}^{2} \\
& \\
&\left.\quad+\zeta_{3}^{2} \otimes N_{4}+\zeta_{3}^{2} \otimes N_{2}^{2}+\zeta_{3}^{2} \otimes N_{1}^{4}+\zeta_{4}^{2} \otimes 1\right]
\end{aligned}
$$


TABLE (continued)

$\varphi_{5}:\left[\zeta_{1}^{2} \otimes N_{5} N_{4}+\zeta_{1}^{2} \otimes N_{5} N_{2}^{2}+\zeta_{1}^{2} \otimes N_{9}+\zeta_{1}^{2} \otimes N_{1} N_{8}+\zeta_{1}^{2} \otimes N_{1} N_{4}^{2}\right.$

$+\zeta_{1}^{2} \otimes N_{1} N_{2}^{2} N_{4}+\zeta_{1}^{2} \otimes N_{1} N_{2}^{4}+\zeta_{1}^{2} \otimes N_{3} N_{6}+\zeta_{1}^{2} \otimes N_{3} N_{2}^{3}$

$+\zeta_{1}^{2} \otimes N_{7} N_{2}$

$+\zeta_{2}^{2} \otimes N_{8}+\zeta_{2}^{2} \otimes N_{1}^{2} N_{6}+\zeta_{2}^{2} \otimes N_{1}^{2} N_{2}^{3}+\zeta_{2}^{2} \otimes N_{3}^{2} N_{2}$

$\left.+\zeta_{3}^{2} \otimes N_{6}+\zeta_{3}^{2} \otimes N_{2}^{3}+\zeta_{3}^{2} \otimes N_{1}^{4} N_{2}+\zeta_{4}^{2} \otimes N_{2}\right]$

$\varphi_{6}:\left[\zeta_{1}^{2} \otimes N_{7} N_{2}^{2}+\zeta_{1}^{2} \otimes N_{1} N_{2}^{2} N_{6}+\zeta_{1}^{2} \otimes N_{1} N_{2} N_{4}^{2}+\zeta_{1}^{2} \otimes N_{3} N_{2}^{2} N_{4}\right.$

$+\zeta_{1}^{2} \otimes N_{1} N_{10}+\zeta_{1}^{2} \otimes N_{3} N_{8}+\zeta_{1}^{2} \otimes N_{11}+\zeta_{1}^{2} \otimes N_{7} N_{4}+\zeta_{1}^{2} \otimes N_{9} N_{2}$

$+\zeta_{1}^{2} \otimes N_{5} N_{6}+\zeta_{1}^{2} \otimes N_{3} N_{2}^{4}+\zeta_{1}^{2} \otimes N_{3} N_{4}^{2}+\zeta_{1}^{2} \otimes N_{5} N_{2}^{3}+\zeta_{1}^{2} \otimes N_{1} N_{2}^{5}$

$+\zeta_{2}^{2} \otimes N_{10}+\zeta_{2}^{2} \otimes N_{1}^{2} N_{2}^{2} N_{4}+\zeta_{2}^{2} \otimes N_{5}^{2}+\zeta_{2}^{2} \otimes N_{1}^{2} N_{2}^{4}$

$+\zeta_{2}^{2} \otimes N_{3}^{2} N_{4}+\zeta_{2}^{2} \otimes N_{3}^{2} N_{2}^{2}+\zeta_{2}^{2} \otimes N_{1}^{2} N_{4}^{2}+\zeta_{2}^{2} \otimes N_{1}^{2} N_{8}$

$+\zeta_{3}^{2} \otimes N_{2}^{2} N_{4}+\zeta_{3}^{2} \otimes N_{4}^{2}+\zeta_{3}^{2} \otimes N_{1}^{4} N_{4}+\zeta_{3}^{2} \otimes N_{1}^{4} N_{2}^{2}+\zeta_{3}^{2} \otimes N_{8}$

$\left.+\zeta_{4}^{2} \otimes N_{2}^{2}+\zeta_{4}^{2} \otimes N_{4}\right]$

$\varphi_{7}:\left[\zeta_{1}^{2} \otimes N_{11} N_{2}+\zeta_{1}^{2} \otimes N_{9} N_{4}+\zeta_{1}^{2} \otimes N_{9} N_{2}^{2}+\zeta_{1}^{2} \otimes N_{1} N_{2}^{6}\right.$

$+\zeta_{1}^{2} \otimes N_{1} N_{2}^{4} N_{4}+\zeta_{1}^{2} \otimes N_{1} N_{6}^{2}+\zeta_{1}^{2} \otimes N_{3} N_{2}^{5}+\zeta_{1}^{2} \otimes N_{3} N_{10}$

$+\zeta_{1}^{2} \otimes N_{7} N_{6}+\zeta_{1}^{2} \otimes N_{5} N_{8}+\zeta_{1}^{2} \otimes N_{1} N_{12}+\zeta_{1}^{2} \otimes N_{1} N_{2}^{2} N_{8}$

$+\zeta_{1}^{2} \otimes N_{3} N_{2}^{2} N_{6}+\zeta_{1}^{2} \otimes N_{3} N_{2} N_{4}^{2}+\zeta_{1}^{2} \otimes N_{5} N_{2}^{2} N_{4}+\zeta_{1}^{2} \otimes N_{5} N_{2}^{4}$

$+\zeta_{1}^{2} \otimes N_{5} N_{4}^{2}+\zeta_{1}^{2} \otimes N_{13}+\zeta_{1}^{2} \otimes N_{1} N_{4}^{3}+\zeta_{1}^{2} \otimes N_{7} N_{2}^{3}$

$+\zeta_{2}^{2} \otimes N_{5}^{2} N_{2}+\zeta_{2}^{2} \otimes N_{1}^{2} N_{2} N_{4}^{2}+\zeta_{2}^{2} \otimes N_{1}^{2} N_{2}^{5}+\zeta_{2}^{2} \otimes N_{1}^{2} N_{10}$

$+\zeta_{2}^{2} \otimes N_{3}^{2} N_{6}+\zeta_{2}^{2} \otimes N_{12}+\zeta_{2}^{2} \otimes N_{3}^{2} N_{2}^{3}+\zeta_{2}^{2} \otimes N_{1}^{2} N_{2}^{2} N_{6}$

$+\zeta_{3}^{2} \otimes N_{2} N_{4}^{2}+\zeta_{3}^{2} \otimes N_{10}+\zeta_{3}^{2} \otimes N_{2}^{2} N_{6}+\zeta_{3}^{2} \otimes N_{1}^{4} N_{6}$

$\left.+\zeta_{3}^{2} \otimes N_{1}^{4} N_{2}^{3}+\zeta_{4}^{2} \otimes N_{2}^{3}+\zeta_{4}^{2} \otimes N_{6}\right]$ 
TABLE (continued)

$$
\begin{aligned}
\varphi_{8}: & \zeta_{1}^{2} \otimes N_{3} N_{2}^{6}+\zeta_{1}^{2} \otimes N_{13} N_{2}+\zeta_{1}^{2} \otimes N_{9} N_{2}^{3}+\zeta_{1}^{2} \otimes N_{9} N_{6} \\
& +\zeta_{1}^{2} \otimes N_{7} N_{2}^{2} N_{4}+\zeta_{1}^{2} \otimes N_{7} N_{8}+\zeta_{1}^{2} \otimes N_{5} N_{10}+\zeta_{1}^{2} \otimes N_{15} \\
& +\zeta_{1}^{2} \otimes N_{7} N_{4}^{2}+\zeta_{1}^{2} \otimes N_{7} N_{2}^{4}+\zeta_{1}^{2} \otimes N_{5} N_{2} N_{4}^{2}+\zeta_{1}^{2} \otimes N_{5} N_{2}^{5} \\
& +\zeta_{1}^{2} \otimes N_{11} N_{2}^{2}+\zeta_{1}^{2} \otimes N_{11} N_{4}+\zeta_{1}^{2} \otimes N_{3} N_{2}^{2} N_{8}+\zeta_{1}^{2} \otimes N_{5} N_{2}^{2} N_{6} \\
& +\zeta_{1}^{2} \otimes N_{3} N_{12}+\zeta_{1}^{2} \otimes N_{1} N_{2}^{4} N_{6}+\zeta_{1}^{2} \otimes N_{1} N_{4}^{2} N_{6}+\zeta_{1}^{2} \otimes N_{1} N_{2}^{2} N_{10} \\
& +\zeta_{1}^{2} \otimes N_{1} N_{2} N_{6}^{2}+\zeta_{1}^{2} \otimes N_{3} N_{4}^{3}+\zeta_{1}^{2} \otimes N_{3} N_{6}^{2}+\zeta_{1}^{2} \otimes N_{1} N_{2}^{7} \\
& +\zeta_{1}^{2} \otimes N_{1} N_{14}+\zeta_{1}^{2} \otimes N_{3} N_{2}^{4} N_{4} \\
& +\zeta_{2}^{2} \otimes N_{1}^{2} N_{12}+\zeta_{2}^{2} \otimes N_{3}^{2} N_{4}^{2}+\zeta_{2}^{2} \otimes N_{3}^{2} N_{2}^{2} N_{4}+\zeta_{2}^{2} \otimes N_{3}^{2} N_{8} \\
& +\zeta_{2}^{2} \otimes N_{5}^{2} N_{4}+\zeta_{2}^{2} \otimes N_{3}^{2} N_{2}^{4}+\zeta_{2}^{2} \otimes N_{5}^{2} N_{2}^{2}+\zeta_{2}^{2} \otimes N_{1}^{2} N_{2}^{4} N_{4} \\
& +\zeta_{2}^{2} \otimes N_{1}^{2} N_{2}^{2} N_{8}+\zeta_{2}^{2} \otimes N_{1}^{2} N_{4}^{3}+\zeta_{2}^{2} \otimes N_{1}^{2} N_{6}^{2}+\zeta_{2}^{2} \otimes N_{1}^{2} N_{2}^{6} \\
& +\zeta_{2}^{2} \otimes N_{14}+\zeta_{2}^{2} \otimes N_{7}^{2} \\
& +\zeta_{3}^{2} \otimes N_{3}^{4}+\zeta_{3}^{2} \otimes N_{1}^{4} N_{4}^{2}+\zeta_{3}^{2} \otimes N_{1}^{4} N_{8}+\zeta_{3}^{2} \otimes N_{1}^{4} N_{2}^{2} N_{4} \\
& +\zeta_{3}^{2} \otimes N_{1}^{4} N_{2}^{4}+\zeta_{3}^{2} \otimes N_{4}^{3}+\zeta_{3}^{2} \otimes N_{2}^{2} N_{8}+\zeta_{3}^{2} \otimes N_{6}^{2}+\zeta_{3}^{2} \otimes N_{12} \\
& +\zeta_{4}^{2} \otimes N_{1}^{8}+\zeta_{4}^{2} \otimes N_{4}^{2}+\zeta_{4}^{2} \otimes N_{2}^{4}+\zeta_{4}^{2} \otimes N_{2}^{2} N_{4}+\zeta_{4}^{2} \otimes N_{8} \\
& \left.+\zeta_{5}^{2} \otimes 1\right]
\end{aligned}
$$

\section{REFERENCES}

[1] J. F. Adams, Stable Homotopy and Generalised Homology, University of Chicago Press, 1974.

[2] V. M. Bŭhstaber, Topological applications of the theory of two-valued formal groups, Math. USSR Izvestija 12 (1978), 177.

[3] V. Gorbunov \& N. Ray, Orientations of Spin bundles and symplectic cobordism, to appear in Publ. RIMS Kyoto University.

[4] H. R. Miller, D. C. Ravenel \& W. S. Wilson, Periodic phenomena in the Adams-Novikov spectral sequence, Annals of Math. 106 (1977), 469-516.

[5] D. C. Ravenel, Complex Cobordism and the Stable Homotopy Groups of Spheres, Academic Press, 1986.

[6] N. Ray, Indecomposables in Tors $\mathrm{MSp}_{*}$, Topology 10 (1971), 261-70.

Manchester University, Manchester, M13 9PL, England.

Current address:

Glasgow University, Glasgow G12 8QW, Scotland.

E-mail address: a.baker@maths.gla.ac.uk 